\title{
EQUIVALENT STATIC WIND LOADS FOR STRUCTURES WITH NON-PROPORTIONAL DAMPING
}

\author{
N. Blaise ${ }^{1}$, T. Canor ${ }^{2}$ and V. Denoël ${ }^{1}$ \\ ${ }^{1}$ Department of Architecture, Geology, Environment and Constructions \\ Structural Engineering Division \\ University of Liege \\ Liege, Belgium \\ E-mail: n.blaise@ulg.ac.be; v.denoel@ulg.ac.be \\ ${ }^{2}$ F.R.S.-FNRS, National Fund for Scientific Research \\ University of Liege \\ Liege, Belgium \\ E-mail: t.canor@ulg.ac.be
}

Keywords: stochastic modal analysis, non-proportional damping, equivalent static wind load, tuned mass damper, high-rise building

\begin{abstract}
In current practice, wind structural design is often carried out using the concept of equivalent static wind loads. The main characteristic of such loadings is to reproduce, with static analyses, the same extreme structural responses as those resulting from a formal buffeting analysis. This paper proposes a method for the computation of equivalent static wind loads for structures with slight non-proportional damping in a modal framework. Because of the smallness of the out-of diagonal terms, this method is based on recent developments related to asymptotic expansion of the modal transfer matrix of such structures. As a main benefit, the static loading is described as a perturbation of the equivalent loading that would be obtained for the uncoupled system. The main contribution of this paper is to formalize the expression of the correction terms resulting from the non-proportionality of damping. The method is presented with a detailed illustrative example.
\end{abstract}

\section{INTRODUCTION}

Originally, Davenport [1] introduced the notion of Equivalent Static Wind Load (ESWL) as a static loading reproducing the same extreme structural responses resulting from a formal buffeting analysis. The structural responses may refer to deflections, internal forces, stresses or any other type of response obtained by linear combinations of the structural displacements. Over the last four decades, methods have been derived to compute ESWL for structures with quasi-static [2, 3] and resonant [4-7] behaviours. Based on the relative proportion of the quasi-static and resonant contributions, the ESWL may be derived in nodal, modal or mixed nodal-modal bases. In this paper, the structural analysis is performed in the full modal basis and the static loadings are defined as weighted combinations of the inertial loads. With each 
mode, an inertial load is associated such that its static application to the structure returns the mode shape. The control of dynamic response of wind-sensitive structures such as wide-span roofs or bridges and high-rise buildings may be obtained using damping devices, e.g. Tuned Mass Damper (TMD) as well as tuned liquid dampers. These damping devices are source of non-proportional damping and consequently, the modal damping matrix and the modal transfer matrix are no longer diagonal. In this case, the dynamic analysis in the modal basis enables to reduce the size of the system, $M$ modes $\ll N$ degrees of freedom, but not to decouple the equations of motion in the modal basis. Lord Rayleigh [8] proposed a decoupling approximation that neglects these off-diagonal elements of the modal damping matrix due to their relative smallness compared with the diagonal ones. If this approximation can not be stated [9], more rigorous techniques must be used. Recently, a method based on an asymptotic expansion of the modal transfer matrix has been proposed in a deterministic framework [10] and then extended to a stochastic context [11]. With this method, full transfer matrix inversions are avoided for all frequencies. For structure with non-proportional damping, this paper aims at the formulation of the asymptotic expansion of the weighting coefficients necessary to derive equivalent static wind loads from the inertial forces. The proposed method is formulated using the by-products resulting from the application of the asymptotic expansion of the modal transfer matrix.

The method is illustrated with a realistic example. A detailed analysis of the asymptotic convergence of both modal amplitudes and weighting coefficients of the inertial forces is realized.

\section{BUFFETING WIND ANALYSIS}

We consider a stationary Gaussian random loading $\mathbf{p}_{t o t}(t)$, representing wind actions, although the concepts could be generalized to other loadings. For convenience the loading is split into a mean part $\overline{\mathbf{P}}$ and a fluctuating part $\mathbf{p}(t)$

$$
\mathbf{p}_{t o t}=\overline{\mathbf{P}}+\mathbf{p}
$$

The motion $\mathbf{x}(t)$ of a linear structure loaded by this random excitation, in the nodal basis, is the solution of the equation of motion

$$
\mathbf{M} \ddot{\mathbf{x}}+\mathbf{C}_{t} \dot{\mathbf{x}}+\mathbf{K x}=\mathbf{p} ; \mathbf{C}_{t}=\mathbf{C}_{s}+\mathbf{C}_{c}+\mathbf{C}_{a}
$$

where $\mathbf{M}, \mathbf{C}_{t}$ and $\mathbf{K}$ are $N \times N$ mass, total damping and stiffness matrices, respectively. The contributions $\mathbf{C}_{s}, \mathbf{C}_{c}$ and $\mathbf{C}_{a}$ are structural, concentrated (due to damping devices) and aerodynamic damping matrices, respectively. Some selected structural responses $\mathbf{r}(t)$ are considered here to be expressed as linear combinations of the nodal displacements

$$
\mathbf{r}=\mathbf{O} \mathbf{x}
$$

where $\mathbf{O}$ is a matrix of influence coefficients, typically known from a finite element model. The structural response of a given linear system can be computed using a small number $M$ of normal modes of vibrations $(M \ll N)$ characterized by mode shapes $\boldsymbol{\Phi}$ [12]. These modes are normalized to have unit generalized masses. Using the modal superposition principle, the equation of motion (2) projected in the modal basis reads

$$
\ddot{\mathbf{q}}+\mathbf{D} \dot{\mathbf{q}}+\mathbf{\Omega q}=\mathbf{g}
$$

where $\mathbf{q}(t)(\mathbf{x}=\mathbf{\Phi q})$ is the vector of modal coordinates, $\mathbf{g}(t)=\boldsymbol{\Phi}^{T} \mathbf{p}(t)$ is the vector of generalized forces, $\boldsymbol{\Omega}$ is a diagonal matrix containing generalized stiffnesses (equal, in this case, to the squared circular frequencies) and $\mathbf{D}=\boldsymbol{\Phi}^{T} \mathbf{C}_{t} \boldsymbol{\Phi}$ is the modal damping matrix. The 
frequency domain formulation of the equation of motion in the modal basis is obtained by a side-by-side Fourier Transform of (4)

$$
\mathbf{Q}=\mathbf{H} \mathbf{G}
$$

where $\mathbf{Q}(\omega)$ and $\mathbf{G}(\omega)$ are respectively the Fourier transforms of $\mathbf{q}(t)$ and $\mathbf{g}(t)$. The modal transfer matrix $\mathbf{H}(\omega)$ is defined by

$$
\begin{aligned}
\mathbf{H} & =\left(\boldsymbol{\Omega}-\omega^{2} \mathbf{I}+i \omega\left(\mathbf{D}_{d}+\mathbf{D}_{o}\right)\right)^{-1} \\
& =\left(\mathbf{I}+\mathbf{H}_{d} \mathbf{J}_{o}\right)^{-1} \mathbf{H}_{d}
\end{aligned}
$$

where $\mathbf{D}_{d}$ and $\mathbf{D}_{o}$ collect the diagonal and off-diagonal elements of $\mathbf{D}$, respectively and $\mathbf{J}_{o}(\omega)=i \omega \mathbf{D}_{o}$. The modal transfer matrix with the decoupling approximation is defined by

$$
\mathbf{H}_{d}=\left(\mathbf{\Omega}-\omega^{2} \mathbf{I}+i \omega \mathbf{D}_{d}\right)^{-1} .
$$

Because the quantity $\left(\mathbf{H}_{d} \mathbf{J}_{0}\right)$ has a small spectral radius [10], an asymptotic expansion of the modal transfer matrix is advantageously considered. It reads

$$
\mathbf{H}_{k}=\mathbf{H}_{d}+\sum_{i=1}^{k}(-1)^{i}\left(\mathbf{H}_{d} \mathbf{J}_{o}\right)^{i} \mathbf{H}_{d}
$$

where $k$ is the approximation order of the transfer function. The structure is further analysed using a spectral approach. The Power Spectral Density (PSD) matrix of the modal coordinates $\mathbf{S}^{(q)}(\omega)$, is obtained by pre- and post-multiplication of the PSD matrix of the generalized Gaussian forces $\mathbf{S}^{(g)}(\omega)$ by the modal transfer matrix,

$$
\mathbf{S}^{(q)}=\mathbf{H} \mathbf{S}^{(g)} \mathbf{H}^{*}
$$

where the superscript ${ }^{*}$ denotes the conjugate transpose operator. The $k^{\text {th }}$ approximation of the PSD matrix of the modal displacements (9) is expressed by

$$
\mathbf{S}^{\left(q_{k}\right)}=\mathbf{S}^{\left(q_{d}\right)}+\sum_{i=1}^{k} \Delta \mathbf{S}^{\left(q_{i}\right)} ; \mathbf{S}^{\left(q_{d}\right)}=\mathbf{H}_{d} \mathbf{S}^{(g)} \mathbf{H}_{d}^{*}
$$

where $\mathbf{S}^{\left(q_{d}\right)}(k=0 \rightarrow k \equiv d)$ is the PSD matrix in the uncoupled system. The successive correction terms $\Delta \mathbf{S}^{\left(q_{i}\right)}$ are expressed in a general recurrence relation

$$
\begin{aligned}
\Delta \mathbf{S}^{\left(q_{1}\right)} & =-\left(\mathbf{H}_{d} \mathbf{J}_{o} \mathbf{S}^{\left(q_{d}\right)}+\mathbf{S}^{\left(q_{d}\right)} \mathbf{J}_{o}^{*} \mathbf{H}_{d}^{*}\right) \\
\Delta \mathbf{S}^{\left(q_{i>1}\right)} & =-\left(\mathbf{H}_{d} \mathbf{J}_{o} \Delta \mathbf{S}^{\left(q_{i-1}\right)}+\Delta \mathbf{S}^{\left(q_{i-1}\right)} \mathbf{J}_{o}^{*} \mathbf{H}_{d}^{*}\right)-\mathbf{H}_{d} \mathbf{J}_{o} \Delta \mathbf{S}^{\left(q_{i-2}\right)} \mathbf{J}_{o}^{*} \mathbf{H}_{d}^{*}
\end{aligned}
$$

with $\Delta \mathbf{S}^{\left(q_{0}\right)}=\mathbf{S}^{\left(q_{d}\right)}$. In the following, the matrix of spectral moments of order $l$ of the modal coordinates, $\mathbf{C}^{l,\left(q_{k}\right)}$, is calculated by integration (along the circular frequencies) of the corresponding PSD matrix

$$
\mathbf{C}^{l,\left(q_{k}\right)}=\int_{-\infty}^{+\infty}|\omega|^{l} \mathbf{S}^{\left(q_{k}\right)} d \omega=\mathbf{C}^{l,\left(q_{d}\right)}+\sum_{i=1}^{k} \Delta \mathbf{C}^{l,\left(q_{i}\right)}
$$

where $k$ denotes one of the aforementioned methods, namely (i) the exact (reference) approach $\left(q_{\infty} \equiv q\right)$, (ii) the decoupling approximation $\left(q_{0} \equiv q_{d}\right)$, (iii) the proposed approximation with $k$ correction terms. Matrix of spectral moments of order $l$ of the structural responses reads

$$
\mathbf{C}^{l,\left(r_{k}\right)}=\boldsymbol{r}^{l,\left(q_{k}\right)} \mathbf{\Upsilon}^{T}
$$


where $\boldsymbol{\Upsilon}=\mathbf{O \Phi}$ is the matrix of modal structural responses. The structural design needs envelope values (minimum and maximum) of the structural responses. The approximation of the envelope $\left(\mathbf{r}^{\min }, \mathbf{r}^{\max }\right)$, while keeping $k$ terms in the asymptotic expansion $(k=0,1,2, \ldots)$ is defined as

$$
\mathbf{r}^{k, \min }=-\mathbf{g}^{r_{k}} \circ \boldsymbol{\sigma}^{r_{k}} ; \mathbf{r}^{k, \max }=\mathbf{g}^{r_{k}} \circ \boldsymbol{\sigma}^{r_{k}}
$$

where $\sigma^{r_{k}}$ and $\mathbf{g}^{r_{k}}$ are the standard deviations and peak factors of the structural responses, respectively and $\circ$ denotes the Hadamard product operator. The peak factor of the $j^{\text {th }}$ structural response is derived from Rice's formula [13]

$$
g_{j}^{r_{k}}=\sqrt{2 \ln n_{j, 0}^{k,+}}+\frac{\gamma}{\sqrt{2 \ln n_{j, 0}^{k,+}}}
$$

where $\gamma=0.5772$ is Euler's constant and $n_{j, 0}^{k,+}$ is the number of zero up-crossings during the observation period with the following formulation

$$
n_{j, 0}^{k,+}=\frac{T}{2 \pi} \sqrt{\frac{C_{j j}^{2,\left(r_{k}\right)}}{C_{j j}^{0,\left(r_{k}\right)}}}
$$

where $\mathrm{T}$ is the observation period taken equal to 10 minutes. For the structural design, the maximum value of the $j^{\text {th }}$ structural response is given by the following formula with a complete quadratic combination approach:

$$
\begin{aligned}
r_{j}^{k, \text { max }} & =g_{j}^{r_{k}}\left(\sum_{m}^{M} \sum_{n}^{M} v_{j m} v_{j n} \sigma_{m n}^{q_{k}}\right)^{1 / 2} \\
& =g_{j}^{r_{k}} \frac{\sum_{m}^{M} \sum_{n}^{M} v_{j m} v_{j n} \sigma_{m n}^{q_{k}}}{\sigma_{j}^{r_{k}}}
\end{aligned}
$$

where $v_{j m}$ is the value of the $j^{\text {th }}$ structural response in the $m^{\text {th }}$ mode and $\sigma_{m n}^{q_{k}}=\sigma_{m n}^{q_{d}}+\sum_{i=1}^{k} \Delta \sigma_{m n}^{q_{i}}$ is the covariance between the $m^{\text {th }}$ and $n^{\text {th }}$ modes formally obtained with (12).

\section{EQUIVALENT STATIC WIND LOAD}

The static analysis under the equivalent static wind load $\mathbf{p}_{j}^{e, k}$ associated with the $j^{\text {th }}$ response reproduces the maximum dynamic response $r_{j}^{k, \max }$, obtained with a classical buffeting analysis, such that

$$
\mathbf{r}_{j}^{e, k}=\mathbf{A} \mathbf{p}_{j}^{e, k} \text { with } r_{j j}^{e, k}=r_{j}^{k, \max }
$$

where $\mathbf{r}_{j}^{e, k}$ is a vector of structural responses under the $j^{t h}$ ESWL and $\mathbf{A}=\mathbf{O K}^{-1}$ is a matrix of influence coefficients. Inertial forces are defined as inertial load excitations that produce the dynamic displacement in (2) under a static analysis, so that

$$
\mathbf{x}=\Phi \mathbf{q} ; \mathbf{K x}=\Psi \mathbf{q}
$$

where $\boldsymbol{\Psi}=\mathbf{K \Phi}$ is the matrix of inertial forces with $\boldsymbol{\psi}_{m}$ the inertial force associated with the $m^{\text {th }}$ modal shape. Chen \& Kareem [6] propose to construct ESWLs as combinations of these inertial forces using weighting coefficients. This paper extends the formulation of these weighting coefficients with correction terms if the system is coupled. The matrix of modal structural responses may be also computed with the inertial forces as

$$
\Upsilon=\mathbf{A} \Psi ; v_{j m}=\mathbf{a}_{j} \psi_{m},
$$


and the introduction of (20) into (17) gives

$$
\begin{aligned}
r_{j}^{k, \text { max }} & =a_{j} g_{j}^{r_{k}} \sum_{m}^{M}\left(\frac{\sum_{n}^{M} v_{j n} \sigma_{m n}^{q_{k}}}{\sigma_{j}^{r_{k}}}\right) \boldsymbol{\psi}_{m} \\
& =a_{j} g_{j}^{r_{k}} \sum_{m}^{M}\left(\frac{\sigma_{j}^{r_{d}}}{\sigma_{j}^{r_{k}}} W_{j m}^{d} \boldsymbol{\psi}_{m}+\sum_{i=1}^{k} \Delta W_{j m}^{i} \boldsymbol{\psi}_{m}\right)
\end{aligned}
$$

where the weighting factors $W_{j m}^{d}$ and $\Delta W_{j m}^{i}$ are defined by

$$
W_{j m}^{d}=\frac{\sum_{n}^{M} v_{j n} \sigma_{m n}^{q_{d}}}{\sigma_{j}^{r_{d}}} ; \Delta W_{j m}^{i}=\frac{\sum_{n}^{M} v_{j n} \Delta \sigma_{m n}^{q_{i}}}{\sigma_{j}^{r_{k}}} .
$$

Comparison of (21) and (18) indicates that the $k^{\text {th }}$ approximation of the static loading $\mathbf{p}_{j}^{e, k}$ is given by the summation of two contributions: (a) a scaling of the equivalent loading that would be obtained if the system was uncoupled $\mathbf{p}^{e, d}$ and (b) a correction $\Delta \mathbf{p}_{j}^{e, k}$, resulting from the non-proportionality of damping, such that

$$
\mathbf{p}_{j}^{e, k}=\alpha_{j}^{k} \mathbf{p}_{j}^{e, d}+\Delta \mathbf{p}_{j}^{e, k}=g_{j}^{k} \sum_{m}^{M} W_{j m}^{k} \boldsymbol{\psi}_{m}
$$

in which

$$
\mathbf{p}_{j}^{e, d}=g_{j}^{d} \sum_{m}^{M} W_{j m}^{d} \boldsymbol{\psi}_{m} ; \Delta \mathbf{p}_{j}^{e, k}=g_{j}^{k} \sum_{m}^{M} \sum_{i=1}^{k} \Delta W_{j m}^{i} \boldsymbol{\psi}_{m}
$$

and where $\alpha_{j}^{k}=\left(g_{j}^{r_{k}} \sigma_{j}^{r_{d}}\right) /\left(g_{j}^{r_{d}} \sigma_{j}^{r_{k}}\right)$ is a scaling coefficient. In other words, the $k^{\text {th }}$ approximation of the weighting coefficients $W_{j m}^{k}$ is given by

$$
W_{j m}^{k}=\alpha_{j}^{k} W_{j m}^{d}+\sum_{i=1}^{k} \Delta W_{j m}^{i}
$$

which well extends the formulation $W_{j m}^{k}=W_{j m}^{d}$ if the modal coupling was neglected. Notice also that $\alpha_{j}^{k}$ degenerates in $\alpha_{j}^{k}=1$ for $k=0$.

\section{ILLUSTRATION}

A $370 \mathrm{~m}$ TV transmission tower is analysed under wind actions. This structure is used in [14] to illustrate the passive control of along-wind response of structures by damping devices. The structural model is a 9-lumped-mass cantilever beam model with 10 nodes and the finite element model is an assembly of classical 2-D beam elements with two DOFs per node (rotation and horizontal displacement). The structural data are reported in Table 1. The mean wind profile is described by a power law

$$
\bar{V}_{i}=\bar{V}_{10}\left(\frac{H_{i}}{10}\right)^{\gamma}
$$

where $\bar{V}_{i}, \bar{V}_{10}, H_{i}$ and $\gamma$ are the mean wind speed of the $i^{t h}$ storey, the mean wind speed at a reference height of 10 meters, the height of the $i^{\text {th }}$ storey and exponent coefficient, respectively. 


\begin{tabular}{|c|r|r|r|r|r|}
\cline { 2 - 6 } \multicolumn{1}{c|}{} & \multicolumn{5}{c|}{ Inter-storey (Element) } \\
\cline { 2 - 6 } \multicolumn{1}{c|}{} & Height & Mass & Stiffness & Damping & Wind area \\
\hline Node Number & $h[\mathrm{~m}]$ & $m\left[10^{3} \mathrm{~kg}\right]$ & $E I\left[10^{8} \mathrm{kN}-\mathrm{m}^{2}\right]$ & $\beta[\mathrm{kN} / \mathrm{m} / \mathrm{s}]$ & $A\left[\mathrm{~m}^{2}\right]$ \\
\hline 1 & 40.0 & 6,134 & $1,162.80$ & 179.92 & 977.8 \\
\hline 2 & 56.0 & 3,853 & 377.80 & 112.89 & 920.6 \\
\hline 3 & 56.0 & 2,578 & 203.74 & 75.54 & 638.6 \\
\hline 4 & 54.5 & 3,032 & 59.30 & 88.82 & 947.6 \\
\hline 5 & 38.5 & 692 & 33.08 & 20.28 & 249.5 \\
\hline 6 & 25.0 & 85 & 8.52 & 2.49 & 150.0 \\
\hline 7 & 35.5 & 72 & 2.13 & 2.11 & 98.2 \\
\hline 8 & 35.5 & 51 & 0.17 & 1.48 & 47.2 \\
\hline 9 & 28.5 & 23 & 0.08 & 0.68 & 20.0 \\
\hline
\end{tabular}

Table 1. Structural data of the $370 \mathrm{~m}$ TV transmission tower. From [14].

A one-dimensional Gaussian velocity turbulence field is considered. The longitudinal turbulent component $v$ of the velocity field is modelled with the spectrum of longitudinal turbulence proposed in [15]

$$
\frac{S_{v}}{\bar{V}_{10}^{2}}=\frac{2 t^{2}}{3|n|\left(1+t^{2}\right)^{4 / 3}}
$$

where $t=1200 n / \bar{V}_{10}$ and $n=\omega /(2 \pi)$. A linearized expression of the applied forces is adopted as

$$
\begin{aligned}
p_{i, t o t} & =\frac{1}{2} \rho_{a} C A_{i}\left(\bar{V}_{i}+v\right)^{2} \\
& \simeq \frac{1}{2} \rho_{a} C A_{i}\left(\bar{V}_{i}^{2}+2 \bar{V}_{i} v\right)=\frac{1}{2} \rho_{a} C A_{i} \bar{V}_{i}\left(\bar{V}_{i}+2 v\right)=\frac{\bar{W}_{10}}{\bar{V}_{10}} C A_{i}\left(\frac{H_{i}}{10}\right)^{\gamma} 2\left(\frac{\bar{V}_{i}}{2}+v\right)
\end{aligned}
$$

where $\rho_{a}, A_{i}, \bar{W}_{10}$ are the air density, surface exposed to wind and mean wind pressure at 10 meters, respectively and $C$ is given by

$$
C=\sqrt{6 K_{0}} C_{a}
$$

in which $K_{0}$ and $C_{a}$ are the surface drag coefficient and drag coefficient, respectively. The PSD matrix of the fluctuating wind pressure reads

$$
S_{i j}^{(p)}=24 K_{0} W_{10}^{2} C_{a}^{2}\left(\frac{H_{i} H_{j}}{100}\right)^{\gamma} A_{i} A_{j} \Gamma_{i j} \frac{1}{4 \pi} \frac{S_{v}}{\bar{V}_{10}^{2}}
$$

where the spanwise coherence function $\Gamma_{i j}$ of $v$ between two points of the tower separated by a height $l$ is modelled by a decreasing exponential

$$
\Gamma_{i j}=\exp \left(-\frac{C_{1}|\omega| l}{2 \pi \bar{V}_{10}}\right)
$$

The aerodynamic data are reported in table 2 .

\begin{tabular}{|c|c|c|c|c|c|c|c|c|}
\hline $\bar{V}_{10}$ & $\bar{W}_{10}$ & $\gamma$ & $Z_{g}$ & $V_{g}$ & $C_{a}$ & $\rho_{a}$ & $C_{1}$ & $K_{0}$ \\
\hline$[\mathrm{m} / \mathrm{s}]$ & {$\left[\mathrm{N} / \mathrm{m}^{2}\right]$} & {$[-]$} & {$[\mathrm{m}]$} & {$[\mathrm{m} / \mathrm{s}]$} & {$[-]$} & {$\left[\mathrm{kg} / \mathrm{m}^{3}\right]$} & {$[-]$} & {$[-]$} \\
\hline 26.41 & 418.5 & 0.15 & 300 & 44 & 0.7 & 1.2 & 7.0 & 0.007 \\
\hline
\end{tabular}

Table 2. Aerodynamic data. From [14]. 


\subsection{Structure without TMD}

We first analyse the structure without the tuned mass damper. Figure 1 depicts the horizontal components of the first four modes and the corresponding inertial forces. The natural frequencies are equal to $0.229,0.348,0.926$ and $1,39 \mathrm{~Hz}$ and the modal dampings are equal to $1.02 \%, 0.67 \%, 0.25 \%$ and $0.17 \%$, respectively.
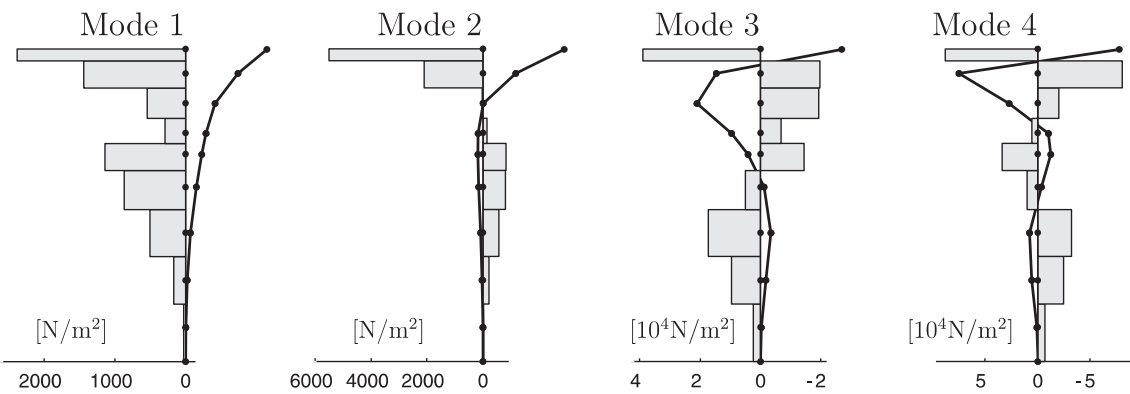

Figure 1: The solid lines with dots depict the first four mode shapes (normalized to a maximum displacement equal to 1 meter) and the grey patches represent the inertial horizontal forces per wind area (i.e. wind pressures).

Equation 2 is first solved with only the structural damping matrix $\mathbf{C}_{s}$. Figure 2-(a,b) illustrates the covariance matrix of spectral moments of order 0 and 2 for the modal coordinates. Mainly, the structure vibrates in its first mode, while the structural velocity requires also the second mode, see Figure 2-(b). The background-to-resonant ratio [16] is a dimensionless modal indicator of the importance of the background component compared to the resonant one. The background-to-resonant ratio in the first mode is equal to 0.22 that indicates that the resonant component is dominating. The index of diagonality [17] defined as $\rho(\mathbf{D})=\sigma\left(\mathbf{D}_{d}^{-1} \mathbf{D}_{o}\right)$, where $\sigma(\cdot)$ is the spectral radius, is an indicator of the diagonal dominance of D. A low value indicates that the off-diagonal terms are small compared to the diagonal ones. In this case, its value is equal to 0.007 and the classical decoupling approximation is applied.


Figure 2: Covariance matrix of order 0 (a) and 2 (b) of the modal coordinates and (c) the weighting coefficients of the inertial forces. For each square, the upper left corner correspond to positive value and the lower right corner to negative value.

Figure 3 illustrates the peak factors $\mathbf{g}^{r_{d}}$ and standard deviations $\boldsymbol{\sigma}^{r_{d}}$ of horizontal deflections (left, $r \equiv x$ ) and bending moments (right, $r \equiv M$ ). 

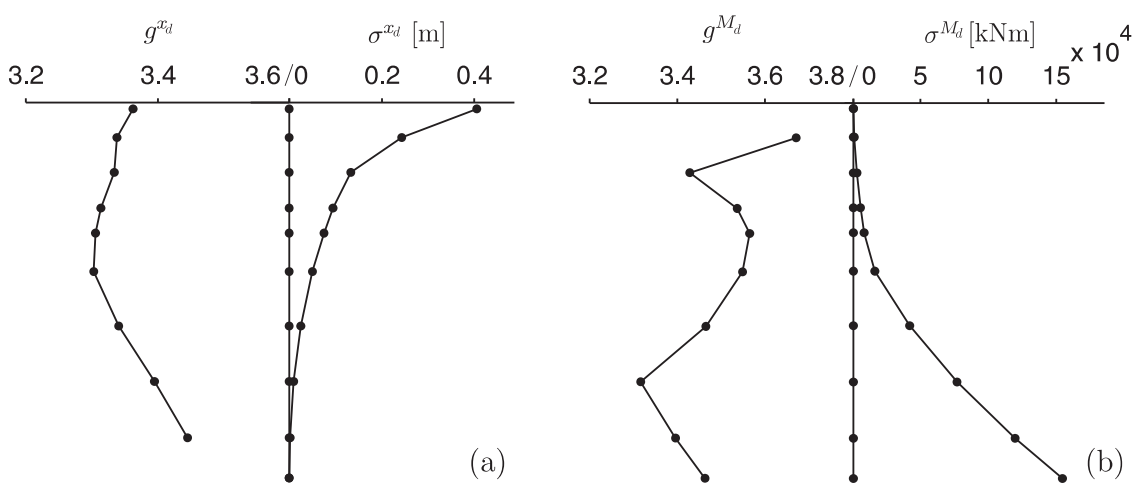

Figure 3: For each graph, on the left the peak factors and on the right the standard deviations of (a) the nodal horizontal displacements and (b) the bending moments, respectively.

For the deflections, peak factors vary from 3.3 to 3.45 and the standard deviation is maximum at the top with a maximum displacement equal to 1.36 meters. For the bending moments, peak factors show larger variations, 3.3 to 3.7. The maximum value at the base is equal to $535 \mathrm{MNm}$. Five specific and representative structural responses are investigated. They are: the bending moment at the base $M_{0}$, the shear force at the base $Q_{0}$, the horizontal displacement at the top $y_{N}$, the rotation at the top $\phi_{N}$ and the horizontal displacement at the fourth level $y_{n}$. Figure 2-(c) shows the weighting coefficients of the inertial forces to produce the equivalent static wind loads represented in Figure 4. As expected, the weighting coefficients take only large values for the first and second modes. The ESWLs for $M_{0}, Q_{0}$ and $y_{n}$ are similar with the same order of magnitude while the ESWLs for $y_{N}$ and $\phi_{N}$ exhibit the largest values for the top two stories.


Figure 4. Equivalent static wind loads for the five specific structural responses.

\subsection{Structure with TMD}

In this section, the response of the structure is mitigated with a tuned mass damper connected to the fourth mass of the structure. Its characteristics are a mass of 494 tons, a stiffness of $1,061 \mathrm{kN} / \mathrm{m}$ and a damping ratio of $17 \%$ based on the minimization of the base moment [14]. The tuned mass damper is tuned to the fundamental frequency of the structure and consequently the first mode is duplicated. Figure 5 depicts the horizontal components of the first five modes with natural frequencies equal to $0.204,0.258,0.352,0.925$, and $1.39 \mathrm{~Hz}$. As a main feature, the tuned mass damper increases significantly the damping ratios of the first two modes to $6.7 \%$ and $10.6 \%$, respectively. 

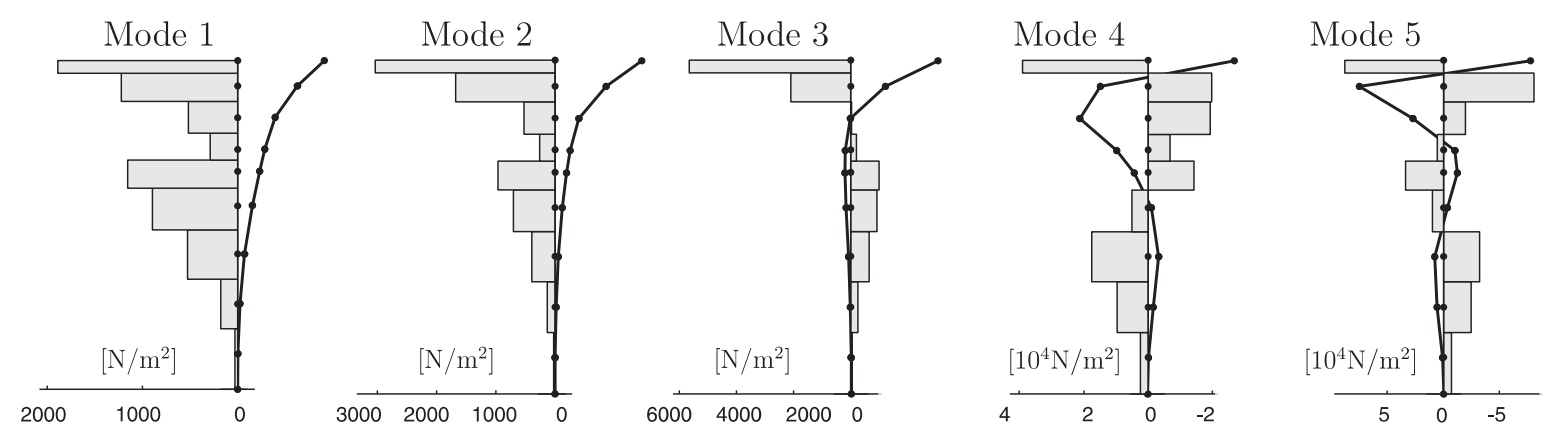

Figure 5: The solid lines with dots depict the first five modal horizontal components (normalized to a maximum displacement equal to 1 meter) and the grey patches represent the inertial horizontal forces per wind area (i.e. wind pressures).

Because of the tuned mass damper, the index of diagonality increases to 1.87 and the classical decoupling approximation can not be formulated anymore. The reference covariance matrix $(l=0$ and $k=\infty)$ of the modal coordinates corresponding to the inversion of the full transfer matrix and the relative error for the successive approximations are represented in Figure 6. The exact background-to-resonant ratio in the first and second mode are equal to 0.75 and 1 respectively. This indicates that thanks to the TMD, the resonant components has been drastically reduced by comparison with the structure without TMD. Figure 6-(a) shows that the third mode has a larger amplitude than the two first which are highly damped. Also, concerning modes 1 and 2 the proximity of their natural frequencies (resp. $0.204 \mathrm{~Hz}$ and $0.258 \mathrm{~Hz}$ ) and the similarities of mode shapes induce dynamic coupling and a high modal correlation coefficient equal to 0.73 . For the three approximations, see Figure 6-(b,c,d), the errors are the most significant for the group of correlated modes (1 and 2). Figure 6-b indicates that neglecting off-diagonal terms of the damping matrix $(k=d)$, induces a maximum error of $30 \%$ on the variance of mode 2 . The proposed method with corrections to the first and second orders allows to reduce this error down to $21 \%$ and $6 \%$, respectively. Therefore with an extension to the second order, the errors are significantly reduced, while remaining maximum for the group of coupled modes.
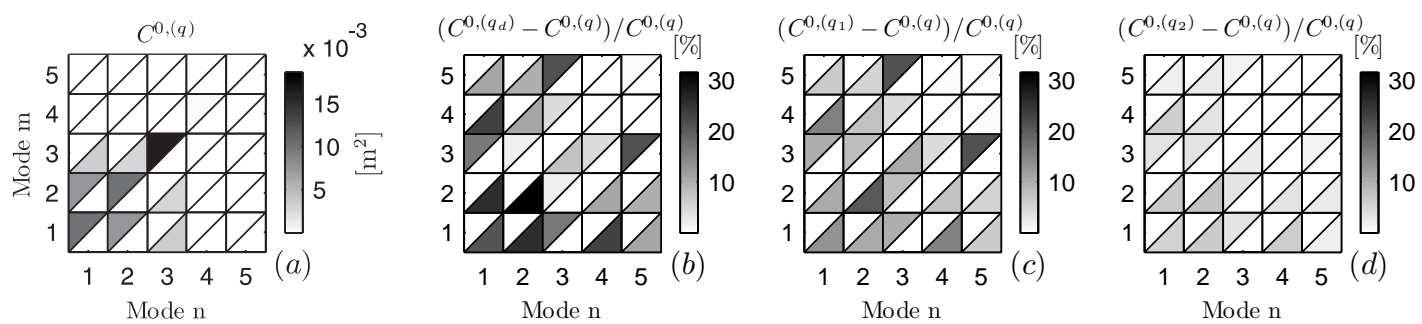

Figure 6: Exact covariance matrix of the modal coordinates (a) $\mathbf{C}^{0,(q)}$ and relative error for different approximations (b) $\mathbf{C}^{0,\left(q_{d}\right)}$, (c) $\mathbf{C}^{0,\left(q_{1}\right)}$ and (d) $\mathbf{C}^{0,\left(q_{2}\right)}$. Relative errors are expressed with respect to $\mathbf{C}^{0,(q)}$.

Reference matrix of spectral moments of order $2(k=\infty)$ for the modal coordinates and the relative error for the different approximations are represented in Figure 7. As previously, the errors are the most significant for the group of correlated modes (1 and 2). The decoupling approximation induces a maximum error of $65 \%$ while the proposed method with an extension to the first and second orders allows to reduce this error down to $36 \%$ and $17 \%$, respectively. This illustrates the common trend that the decoupling approximation generates significant discrepancies on the estimation of structural velocity and acceleration. The proposed method is a simple way to circumvent this inaccuracy. 

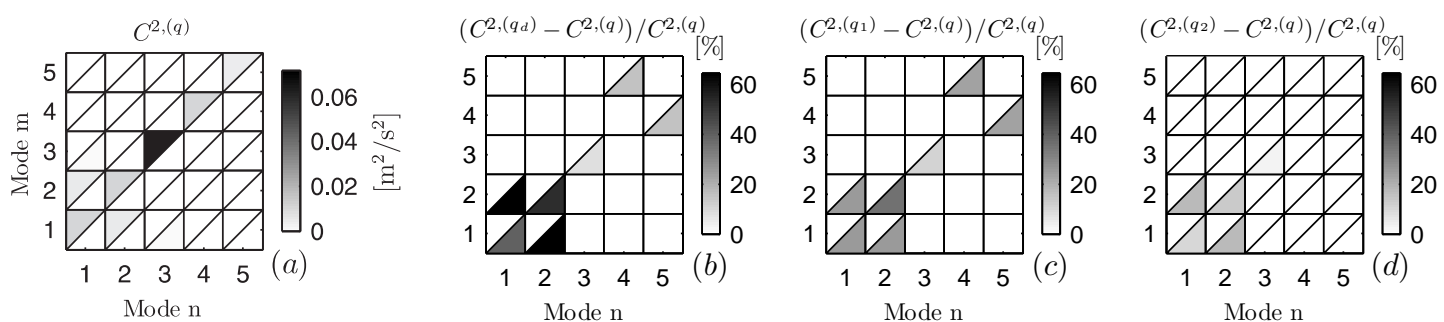

Figure 7: Exact matrix of spectral moments of order 2 for the modal coordinates (a) $\mathbf{C}^{2,(q)}$ and relative error for different approximations (b) $\mathbf{C}^{2,\left(q_{d}\right)}$, (c) $\mathbf{C}^{2,\left(q_{1}\right)}$ and (d) $\mathbf{C}^{2,\left(q_{2}\right)}$. Relative errors are expressed with respect to $\mathbf{C}^{2,(q)}$.

Figure 8 illustrates the reference $(k=\infty)$ peak factors and standard deviations of horizontal deflections and bending moments. For the deflections, peak factors vary from 3.2 to 3.45 and the maximum value at the top has been reduced to 0.72 meters. The maximum bending moment has been also reduced to $356 \mathrm{MNm}$. On the right of each graph is also represented the relative errors corresponding to the decoupling approximation $(k=d)$ and the proposed method with corrections to the first $(k=1)$ and second orders $(k=2)$. The decoupling approximation lead to important underestimations up to $12.5 \%$ for the displacements and $8 \%$ for the bending moments. With only the first correction, these extreme underestimations are divided by two. The second order of approximation provides underestimations less than 3 $\%$ and therefore this order of approximation is used for the computation of equivalent static wind loads. For the decoupling approximation, relative errors on the peak factors are less than $1 \%$ for an error of $10 \%$ on $v_{0}^{+}$. These errors are therefore not represented. Indeed, despite large errors on spectral moments of order 0 and 2, errors committed on $v_{0}^{+}$are lower and finally, variations on peak factors are marginal. Peaks factors obtained with the classical decoupling approximation may be used as a simplification and corrections on matrix of the spectral moments of order 2 are not necessary provided we are interested in structural displacements only.
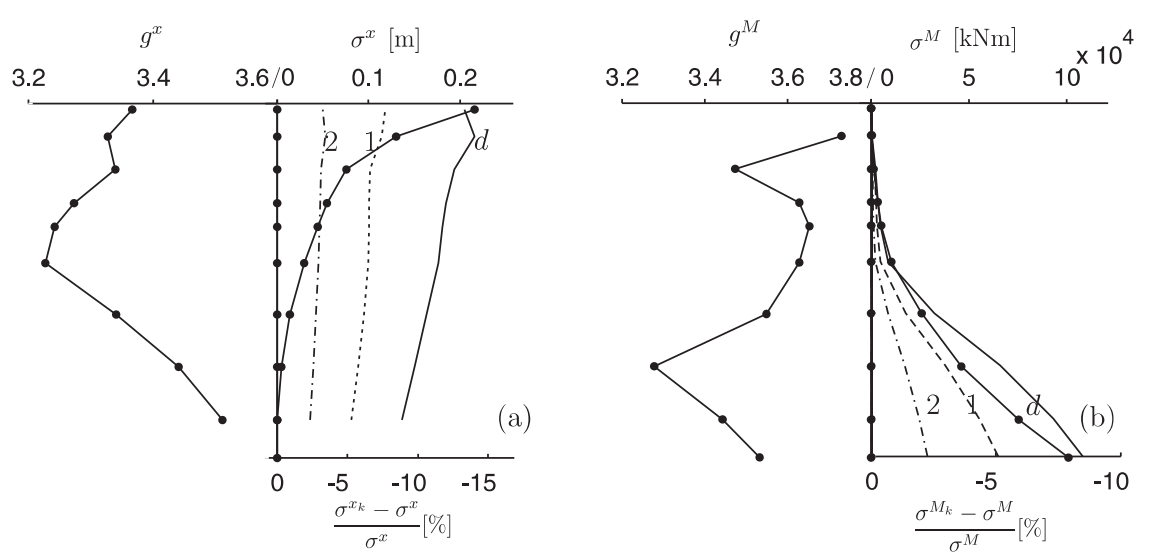

Figure 8: For each graph, on the left the peak factors and on the right the standard deviations for (a) the nodal horizontal displacements and (b) the bending moments, respectively. The upper axis, corresponding to reference values $(k=\infty)$, is associated with lines with black dots. The lower axis, corresponding to relative errors, is associated with solid $(k=0)$, dashed $(k=1)$ and dash-dotted $(k=2)$ lines.

Reference weighting coefficients $\left(W^{\infty}=W\right.$ ) of the inertial forces and the relative errors for the different approximations are represented in Figure 9. As expected, the orders of errors are similar to those reported for the modal amplitudes, see Figure 6. The order of errors for the weighting coefficients in each mode are similar no matter the structural response investigated. 

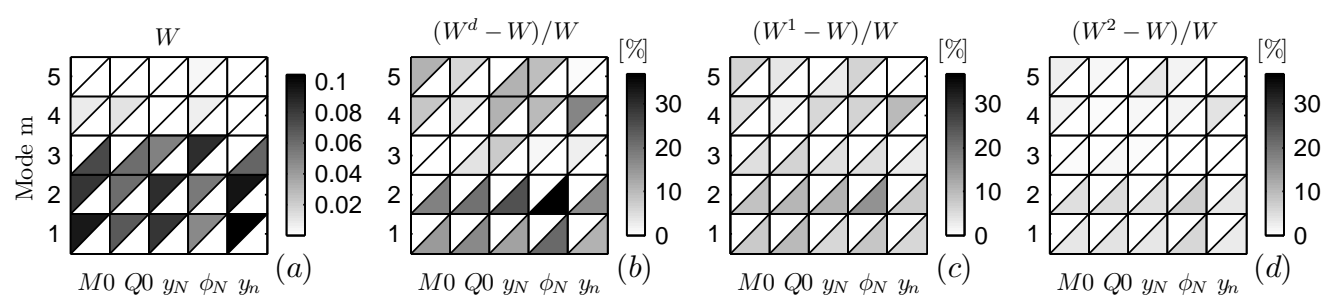

Figure 9: reference weighting coefficients (a) $\mathbf{W}$ and relative error for different approximations (b) $\mathbf{W}^{(d)}$, (c) $\mathbf{W}^{(1)}$ and (d) $\mathbf{W}^{(2)}$. Relative errors are expressed with respect to $\mathbf{W}$.

Figure 10 depicts the reference equivalent static wind loads for the structural responses. Also, the ESWLs obtained with the decoupling approximation and the proposed method with a second order approximation are superimposed. Large over- and under- estimations of the reference loadings occur with the decoupling approximation. The ESWLs resulting from the proposed method with a second order approximation correctly fit the reference static loadings. By comparison with the ESWLs for the structure without TMD, the magnitude of the loadings have been reduced and the distributions have changed.


Figure 10: Reference equivalent static wind loads for the five specific structural responses and those obtained with the decoupling approximation and the proposed method with second order corrections.

\section{CONCLUSIONS}

Wide-span roofs or bridges and high-rise buildings may be excited by the wind loading beyond acceptable level of displacements, internal forces or stresses. For such cases, among other solutions, damping devices are usually used to control and reduce the wind response of these structures. These damping devices may be tuned mass or liquid damper which introduce non-proportional damping. A new method consisting in the asymptotic expansion of the modal transfer matrix enables to avoid full transfer matrix inversion. This work further developed the method for the establishment of equivalent static wind loads for structures with non-proportional damping analysed in the modal basis. As a major finding, the expressions of the asymptotic expansion of the weighting coefficients of inertial forces necessary to establish the ESWL are derived using the by-products of the original method. For the studied case of a $370 \mathrm{~m} \mathrm{TV}$ transmission tower with a tuned mass damper producing an index of diagonality equal to 1.87 , a second order approximation of the modal transfer matrix is sufficient to reduce the errors on the deflections and bending moments to less than $3 \%$ compared to $10 \%$ for the classical decoupling approximation. It has also been shown that peak factors do not vary significantly between the coupled and uncoupled system. For these quantities, the decoupling approximation can be used as a simplification. Finally, large errors on the ESWLs obtained with the decoupling approximation were highlighted while those obtained 
with the proposed method with two corrections terms correctly fit the exact ESWLs. This emphasizes, once more, the reliability of the proposed method.

\section{REFERENCES}

[1] A. G. Davenport. Gust loading factors. In: Proceedings of the American Society of Civil Engineers. Journal of Structural Division, 93(3):11-34, 1967.

[2] J. D. Holmes. Distribution of peak wind loads on a low-rise building. Journal Of Wind Engineering and Industrial Aerodynamics, 29(1-3):59-67, 1988.

[3] M. Kasperski. Extreme wind load distributions for linear and nonlinear design. Engineering Structures, 14(1):27-34, 1992.

[4] J. D. Holmes. Along-wind response of lattice towers - iii. effective load distributions. Engineering Structures, 18(7):489-494, 1996.

[5] Y. Zhou, M. Gu, and H. Xiang. Alongwind static equivalent wind loads and responses of tall buildings. part ii: Effects of mode shapes. Journal of Wind Engineering and Industrial Aerodynamics, 79(1-2):151-158, 1999.

[6] X. Z. Chen and A. Kareem. Equivalent static wind loads for buffeting response of bridges. Journal of Structural Engineering-Asce, 127(12):1467-1475, 2001.

[7] N. Blaise and V. Denoël. Principal static wind loads. Journal of Wind Engineering and Industrial Aerodynamics, 113:29-39, 2013.

[8] J. W. Strutt Lord Rayleigh. The Theory of Sound, volume 1. Dover Publications, New York, 1877, re-issued 1945.

[9] M. Morzfeld, N. Ajavakom, and F. Ma. Diagonal dominance of damping and the decoupling approximation in linear vibratory systems. Journal of Sound and Vibration, 320(1-2):406-420, 2009.

[10] V. Denoël and H. Degée. Asymptotic expansion of slightly coupled modal dynamic transfer functions. Journal of Sound and Vibration, 328(1-2):1-8, 2009.

[11] T. Canor, N. Blaise, and V. Denoël. Efficient uncoupled stochastic analysis with nonproportional damping. Journal of Sound and Vibration, 331(24):5283-5291, 2012.

[12] R. W. Clough and J. Penzien. Dynamics of structures. McGraw-Hill, New-York, 2nd edition edition, 1993.

[13] S.O. Rice. Mathematical analysis of random noise. Bell System Technical Journal, 24:45-156, 1945.

[14] Y. L. Xu, B. Samali, and K. C. S. Kwok. Control of along-wind response of structures by mass and liquid dampers. J. Engineering Mechanics, 118(1):20-39, 1992.

[15] A. G. Davenport. The application of statistical concepts to the wind loading of structures. Proceedings of the Institute of Civil Engineers, 19:449-472, 1961.

[16] V. Denoël. Estimation of modal correlation coefficients from background and resonant responses. Structural Engineering And Mechanics, 32(6):725-740, 2009.

[17] R. A. Horn and C. R. Johnson. Matrix Analysis. Cambridge University Press, Cambridge, United Kingdom, 1945. 